\title{
LA DEMOCRAZIA PARTECIPATIVA E I SUOI LATI OSCURI*
}

\author{
PARTICIPATORY DEMOCRACY AND ITS DARK SIDES
}

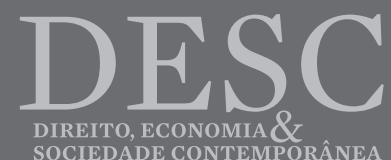

* Il presente intervento riprende il contenuto della relazione "La democrazia partecipativa a livello statale" presentata al Convegno "Rappresentanza e partecipazione tra Stato e Regioni", Regione Umbria-Assemblea legislativa, Centro Studi Giuridici e Politici, Palazzo Cesaroni, Perugia, 8 novembre 2019, nonché il contenuto della pubblicazione, in lingua inglese, Participatory democracy and its dark sides, in Federalismi.it, n. 12/2020. 


\title{
LA DEMOCRAZIA PARTECIPATIVA E I SUOI LATI OSCURI*
}

\author{
PARTICIPATORY DEMOCRACY AND ITS DARK SIDES
}

\section{ALESSANDRA ALGOSTINO \\ Università degli Studi di Torino alessandra.algostino@unito.it https://www.giurisprudenza.unito.it/persone/alessandra.algostino https://orcid.org/0000-0002-5302-1564}

\begin{abstract}
Riassunto:
Nell'intervento si riflette sul concetto di democrazia partecipativa, nella prospettiva del suo inserimento nello spazio di due principi e, insieme, obiettivi da rendere effettivi, attraverso i quali si articola l'essenza della democrazia: la sovranità popolare e la partecipazione.

Si muove da una definizione della locuzione "democrazia partecipativa", mettendo in luce i tratti che la contraddistinguono dalla declinazione "rappresentativa" e "diretta" della democrazia, così come dalla democrazia dal basso. L'intento è proporre una riflessione sull'apporto che la democrazia partecipativa può offrire in direzione di una democrazia quanto più possibile effettiva, senza misconoscere i rischi che essa presenta e nella consapevolezza della strutturale dinamicità, tensione e incompiutezza della democrazia.
\end{abstract}

Parole-chiavi: Democrazia partecipativa, Sovranità popolare, Partecipazione, Cittadinanza, Pluralismo

\footnotetext{
Abstract:

The essay reflects on the concept of participatory democracy, in the perspective of its insertion into the space of two principles and, together, objectives to be made effective, through which the essence of democracy is articulated: popular sovereignty and participation.

It starts from a definition of the term "participatory democracy", highlighting the traits that distinguish it from the "representative" and "direct" declination of democracy, as well as from democracy from below. The intent is to propose a reflection on the contribution that participatory democracy can offer in the direction of a democracy that is as effective as possible, without ignoring the risks it presents and in the awareness of the structural dynamism, tension and incompleteness of democracy.

Parole-chiavi: Participatory democracy, Popular sovereignty, Participation, Citizenship, Pluralism
} 


\section{Una imprescindibile premessa: sul senso della sovranità popolare e della} partecipazione

Senza pretesa di approfondire il concetto di sovranità popolare, o di ricostruire le teorie sulla partecipazione, ma al più limitato scopo di individuare il terreno sul quale costruire un discorso sulla democrazia partecipativa, conviene muovere dal riconoscimento della sovranità popolare, ovvero, nell'orizzonte della Costituzione italiana, dall'art. 1, co. 2, e dall'obiettivo della «effettiva partecipazione» («di tutti i lavoratori all’organizzazione politica, economica e sociale del Paese», art. 3, co. 2, Cost.).

La sovranità popolare rappresenta un prius rispetto allo Stato, è il fondamento dello Stato, è oggetto, detto altrimenti, di una semplice constatazione ${ }^{1}$; in quanto tale, logicamente, non si identifica con lo Stato $^{2}$. Lo Stato e le sue istituzioni sono espressione, in un ordinamento democratico, di sovranità popolare ${ }^{3}$, nel senso che lo «Stato-soggetto» assume, rispetto al principio della sovranità popolare, «carattere strumentale» (Crisafulli) ${ }^{4}$, ma non esauriscono la sovranità popolare.

La sovranità popolare - si può aggiungere - trova un canale privilegiato di espressione nella democrazia rappresentativa: si pensi all'esercizio di sovranità attraverso i diritti politici, sia che siano intesi in senso stretto come diritti elettorali sia in senso ampio come comprendenti anche la partecipazione attraverso i partiti politici ${ }^{5}$. Ma, ponendosi nel solco della tesi di Crisafulli, anche l'esercizio del diritto di riunione o della libertà di associazione ricadono nell'alveo della sovranità popolare e costituiscono una forma di partecipazione ${ }^{6}$. L'esercizio dei diritti fondamentali, in altri termini, citando Ferrajoli, può configurarsi come esercizio di sovranità popolare frammentata: «i diritti fondamentali danno forme e contenuti alla volontà popolare» e costituiscono «frammenti di sovranità popolare in capo a tutti e a ciascun cittadino» ${ }^{7}$.

1 Cfr. G. FERRARA, La sovranità popolare e le sue forme, in S. LABRIOLA (a cura di), Valori e principi del regime repubblicano, 1.I Sovranità e democrazia, Laterza, Roma-Bari, 2006, spec. pp. 261-262.

2 ... come invece parte della dottrina, Tosato ad esempio, ha sostenuto (E. TOSATO, Sovranità del popolo e sovranità dello Stato, in Riv. Trim. Dir. Pubbl., 1957).

3 Sul legame democrazia-sovranità popolare, per tutti, cfr. C. MORTATI, Istituzioni di diritto pubblico, I, X ed. rielaborata ed aggiornata a cura di F. Modugno, A. Baldassarre e C. Mezzanotte, Cedam, Padova, 1991, p. 153.

4 V. CRISAFULLI, La sovranità popolare nella Costituzione italiana, in Rassegna Giuliana di Diritto e Giurisprudenza, 1954 , p. 57.

5 Se si identifica la «sovranità» con l'esercizio dei diritti di decisione (voto) spettanti al corpo elettorale, il «popolo» titolare della sovranità viene ad essere identificato con il corpo elettorale: così C. MORTATI, Art. 1, in G. BRANCA (a cura di), Commentario della Costituzione, Principi fondamentali, Bologna-Roma, 1957, pp. 27-28; mentre, in senso critico sulla coincidenza fra corpo elettorale e popolo, cfr. V. CRISAFULLI, La sovranità popolare, cit., spec. pp. 26 ss.

$6 \mathrm{Si}$ adotta la concezione che contempla, tra le forme che rendono effettivo l'esercizio della sovranità popolare, l'associarsi ad un partito, concorrendo a determinare la politica nazionale, o l'esercitare il diritto di riunirsi pacificamente e senz'armi, o l'associarsi liberamente per fini che non siano vietati ai singoli dalla legge penale (T. MARTINES, Art. 56-58, in G. BRANCA (a cura di), Commentario della Costituzione, Le Camere, Tomo I, Bologna-Roma, 1984, p. 72; per tutti, inoltre, in tal senso, cfr. V. CRISAFULLI, La sovranità popolare, cit., in particolare pp. 40 ss.; diversamente C. MORTATI, Art. 1, cit., pp. 27-28, e D. NOCILLA, Popolo (dir. cost.), in Enc. dir., XXXIV, Giuffrè, Milano, 1985, p. 387).

7 L. FERRAJOLI, Principia iuris. Teoria del diritto e della democrazia, 2. Teoria della democrazia, Laterza, Roma.Bari, 2007, pp. $10-11$. 
È necessario però un passo ulteriore: diritto di voto, partiti, associazioni e libertà, sono esplicitamente contemplati e tutelati dalla Costituzione ${ }^{8}$; possono considerarsi espressione di sovranità popolare anche manifestazioni non formalizzate?

Nel senso di una risposta affermativa militano (quantomeno) due argomentazioni.

La prima. La sovranità popolare può manifestarsi in forme non predeterminate proprio in quanto essa costituisce espressione dell'esercizio di diritti, come il diritto di riunione, la libertà di associazione, la libertà di manifestazione del pensiero ${ }^{9}$.

La seconda. La nozione di sovranità popolare si accompagna ad un concetto ampio di partecipazione $^{10}$, che riflette le diverse facce che compongono la democrazia (sostanziale) politica, sociale, economica - che trovano una traduzione letterale nelle tre dimensioni della formula «organizzazione politica, economica e sociale» di cui all'art. 3, co. 2, Cost.

L'art. 3, co. 2, Cost., cioè, ha le sue radici nell'art. 1 Cost $^{11}$., e si irradia nelle norme in tema di rapporti economici, così come nelle libertà politiche e nei diritti sociali12 ${ }^{12}$ la partecipazione effettiva gioca nei vari ambiti un ruolo dinamico, esprimendo la tensione che è insita nel processo democratico ${ }^{13}$.

Il carattere attivo e la declinazione come «effettiva» della partecipazione inducono, non solo a ritenere legittime, bensì anche a valorizzare esperienze nuove di partecipazione, che conoscano o meno una istituzionalizzazione. Il riferimento è, per l'appunto, alla democrazia partecipativa ${ }^{14}$, ma anche alla democrazia come attività di "surveillance" (Rosanvallon) o alla democrazia dal basso (nella pluralità delle manifestazioni ad essa riconducibili, dall'esperienza delle "fabbriche recuperate" ai movimenti territoriali o per il diritto alla casa, da Fridays For Future ai centri sociali, etc.).

Sempre dal radicamento della partecipazione nell'art. 3, co. 2, Cost., si può poi dedurre il suo stretto legame con le istanze di giustizia sociale: da un lato, la partecipazione necessita delle pre-condizioni assicurate attraverso la liberazione dagli «ostacoli di ordine economico e sociale» (ponendosi rispetto a questi ultimi come un obiettivo); dall'altro, essa costituisce strumento del progetto di emancipazione individuale e sociale. La partecipazione, in altri

8 Rispettivamente ai sensi degli articoli 49 e 18, ma anche, in via generale, ai sensi dell'articolo 2, della Costituzione.

9 Cfr. U. ALLEGRETTI, Il Movimento internazionale come attore costituzionale, in Dem. e dir., n. 1/2004, pp. 68 ss.

10 Vedi V. CRISAFULLI, La sovranità popolare, cit., p. 57; recentemente, cfr. S. D’ALBERGO, Dalla democrazia sociale alla democrazia costituzionale (un percorso dell'ideologia giuridica), in Costituzionalismo.it, n. 3/2005, p. 10; G. AZZARITI, Democrazia partecipativa: cultura giuridica e dinamiche istituzionali, in Costituzionalismo.it, n. 3/2009; M. MANETTI, Costituzione, partecipazione democratica, populismo, in Rivista AIC, n. 3/2018, p. 389.

11 In argomento, sul nesso fra lavoro, partecipazione, uguaglianza, ex multis, cfr. M. DOGLIANI, C. GIORGI, Costituzione italiana: art. 3, Carocci, Roma, 2017, p. 101; M. LUCIANI, Radici e conseguenze della scelta repubblicana di fondare la Repubblica democratica sul lavoro, in ADL - Argomenti dir. lavoro, 3/2010, pp. $634 \mathrm{~s}$.

12 L. BASSO, Per uno sviluppo democratico nell'ordinamento costituzionale italiano, in Studi per il ventesimo anniversario dell'Assemblea costituente, v. IV: Aspetti del sistema costituzionale, Firenze, 1969, p. 17.

13 Cfr. B. CARAVITA, Oltre l'eguaglianza formale. Un'analisi dell'art. 3 comma 2 della Costituzione, Padova, 1984, p. 113.

14 Per interessanti considerazioni sul legame fra democrazia partecipativa e art. 3, co. 2, Cost., cfr. A. VALASTRO, Gli istituti di partecipazione fra retorica delle riforme e umiltà dell'attuazione, in Costitutizonalismo.it, n. 1/2017, spec. pp. 59 ss.; M. PICCHI, Il diritto di partecipazione: note preliminari (per l'effettività dei diritti sociali), nella rivista del Gruppo di Pisa, n. 3/2012. 
termini, si pone allo stesso tempo come oggetto e come soggetto del progetto di trasformazione sociale e di costruzione di una democrazia sostanziale. Se un limite si vuol individuare, esso appare intrinseco al concetto di partecipazione democratica.

Si muove, dunque, da un concetto di sovranità popolare, interpretato come aperto e dinamico, in stretta connessione con la «partecipazione effettiva» concepita come elemento di vitalità della democrazia, nella prospettiva - citando Rosa Luxemburg - che "“il faticoso movimento delle istituzioni democratiche" possiede un potente correttivo... nel vivente movimento delle masse, nella loro pressione ininterrotta» ${ }^{15}$, che può dar luogo a nuove forme di partecipazione, quale la democrazia partecipativa ${ }^{16}$.

\section{Il quid distintivo della democrazia partecipativa}

La locuzione "democrazia partecipativa" si afferma negli anni Sessanta e Settanta, nell'ambito di quella che Held definisce Nuova Sinistra ${ }^{17}$.

Si ritiene che la partecipazione - riprendendo le parole della Pateman - sia capace, al netto delle realistiche considerazioni sull'effettivo coinvolgimento dell' «ordinary man», di rendere ciascuno «meglio in grado di valutare le prestazioni dei rappresentanti a livello nazionale», «meglio attrezzato per prendere decisioni», nonché più preparato ad esercitare un controllo sulla propria vita e sul proprio ambiente ${ }^{18}$. In questa prospettiva si sostiene l'estensione della partecipazione a «sfere al di fuori del governo statale», in primo luogo quella «dell'impresa» ${ }^{19}$ , ma anche della comunità locale, nell'ambito di un «sistema istituzionale aperto per assicurare la possibilità di sperimentare nuove forme politiche $»^{20}$.

La narrazione della democrazia partecipativa oggi prevalente riprende alcune caratteristiche di quella sintetizzata; ne assume il portato educativo e la capacità di rinnovare la democrazia. Entra, inoltre, in alcune costituzioni: si pensialla qualificazione come «partecipativo» del carattere democratico dello Stato nella Costituzione venezuelana o all'esplicito riferimento alla democrazia partecipativa nella Costituzione boliviana ${ }^{21}$, ma anche alla Costituzione

15 R. LUXEMBURG, La rivoluzione russa, 1918.

16 Connette all'incompiutezza della democrazia e alla sua essenza dinamica l'emergere di strumenti relativi alla democrazia partecipativa, nell'habermasiano "spazio pubblico politico", U. ALLEGRETTI, Democrazia partecipativa e processi di democratizzazione, in Dem. e dir., n. 2/2008, pp. 177-178.

17 D. HELD, Models of Democracy, 1996, trad. it. Modelli di democrazia, il Mulino, Bologna, 1997, pp. 369 ss.

18 C. PATEMAN, Participation and democratic theory, Cambridge University Press, 1970, p. 110.

19 C. PATEMAN, Participation and democratic theory, cit., pp. 106-111.

20 D. HELD, Modelli di democrazia, cit., p. 379.

21 Si veda la Costituzione bolivariana del Venezuela, 1999, che nel Preambolo si riferisce al «fin supremo de refundar la República para establecer una sociedad democrática, partecipativa...», nell'art. 2 definisce il Venezuela un «Estado democrático y social de Derecho y de Justicia» e all'art. 6 specifica che «el gobierno de la República Bolivariana de Venezuela y de las entidades políticas que la componen es y será siempre democrático, partecipativo...» (in argomento, per un primo commento, cfr. D. PICCIONE, Presidenzialismo e democrazia partecipativa nella Costituzione bolivariana del Venezuela, in Dir. pubbl. comp. ed europeo, n. 2005-I, pp. 3 ss.; G. ALLEGRETTI, Politiche di partecipazione in Venezuela: tra discorso costituzionale e pratiche sperimentali, in Dem. e dir., n. 3/2006, pp. 42 ss., e ID., Politiche di partecipazione in Venezuela: l'inizio di un percorso di statuizione normativa, in Dem. e dir., $n$. 4/2006, pp. 27 ss.); o la Nueva Constitución política del Estado della Bolivia, 2007, che, all'art. 11, sancisce: «el Estado adopta para su gobierno la forma democrática participativa, representativa y comunitaria». 
portoghese, che, all'art. 2, prevede il rafforzamento della democrazia partecipativa.

Nelle esperienze latinoamericane, a differenza di quelle europee, è forte il legame fra democrazia partecipativa e istanze di giustizia sociale, redistribuzione delle ricchezze e lotta alla diseguaglianza ${ }^{22}$.

Nelle versioni europee odierne, si perde, segnando la distanza rispetto alle teorie degli anni Sessanta, l'ambizione a rendere la democrazia partecipativa il volano per una democratizzazione di altre sfere, quali, in primis, quelle delle relazioni industriali ed economica. Qui il concetto più à la page è "governance", la quale - scusandomi per il carattere assertivo, ma ai fini del presente lavoro non pare necessario approfondire oltre il concetto - costituisce non tanto una sede di partecipazione della società civile, quanto una procedura impositivo-concertativa, che, dietro la retorica mistificatrice di una tavola rotonda alla quale siedono i potenziali interessati (con un mélange fra soggetti pubblici e soggetti privati), riproduce le diseguaglianze e mira a disinnescare il potenziale rivendicativo del quale possono essere portavoce settori della società civile ${ }^{23}$.

I meccanismi di democrazia partecipativa si attestano all'interno del campo istituzionale, configurandosi come strumento di gestione del governo, che sia a livello locale o nazionale.

Si perde, cioè, l'immagine della democrazia partecipativa come luogo di sperimentazione di forme di autogestione, ovvero di forme politiche alternative, e di essa si afferma una versione che si può definire "interna" rispetto alle istituzioni, in funzione integrativa rispetto al processo decisionale politico-amministrativo ${ }^{24}$.

La domanda, allora, è: quanto il fatto che si discorra di modalità di organizzazione del potere sposta il focus dal diritto di partecipazione, nella prospettiva del coinvolgimento dei cittadini, in chiave emancipante, alla governabilità ${ }^{25}$, inscritta in una logica decisionista, nel segno dell'efficacia della decisione?

Per rispondere è necessario addentrarsi nel terreno della democrazia partecipativa, delle sue pratiche, dei suoi rapporti con le altre declinazioni della democrazia; e preliminare si rivela la ricerca di una sua definizione.

Nella molteplicità ed eterogeneità dei significati e delle figure che la democrazia

22 U. ALLEGRETTI, La democrazia partecipativa in Italia e in Europa, in Rivista AIC, n. 1/2011, p. 5.

23 In tema, ex plurimis, M.R. FERRARESE, La governance tra politica e diritto, il Mulino, Bologna, 2010; A. ARIENZO, Dalla corporate governance alla categoria politica di governance, in G. BORRELLI (a cura di), Governance, Dante\&Descartes, Napoli, 2004, pp. 125 ss.; G. MESSINA, Diritto liquido? La governance come nuovo paradigma della politica e del diritto, Franco Angeli, Milano, 2012; nonché, anche per ulteriori indicazioni bibliografiche, A. ALGOSTINO, Democrazia, rappresentanza, partecipazione. Il caso del movimento No Tav, Jovene, Napoli, 2011, pp. 122 ss.

24 P. ROSANVALLON, La légitimité démocratique. Impartialité, réflexivité, proximité, Seuil, Paris, 2008, p. 323, evidenzia, al di là dell'utilizzo della stessa terminologia, la differenza fra le esperienze di democrazia partecipativa "tradizionali" e la maggior parte di quelle attuali, individuandola innanzitutto nell'origine istituzionale delle seconde, nel loro carattere non sostitutivo di precedenti meccanismi di decisione e nella loro limitazione ad alcuni campi particolari; il che induce a leggerle come strumenti di governo più che di autogestione.

25 In senso critico, sulla governabilità come tecnica coattiva, cfr. G. FERRARA, La crisi del neoliberismo e della governabilità coatta, in Costituzionalismo.it, n. 1/2013). 
partecipativa assume ${ }^{26}$, un suo primo connotato emerge a contrario, distinguendola sia dalla democrazia rappresentativa sia dalla democrazia diretta classica ${ }^{27}$ : essa è una forma di coinvolgimento dei $\operatorname{cittadini}^{28}$ non riconducibile al circuito elettorale-rappresentativo o referendario ${ }^{29}$; la mancanza degli elementi della spontaneità, dell'auto-organizzazione e dell'indipendenza rispetto alle istituzioni, segna invece la sua differenza rispetto alla democrazia dal basso ${ }^{30}$.

Esperienze di democrazia partecipativa sono, per intendersi, i bilanci partecipativi ${ }^{31}$, le giurie civiche berlinesi ${ }^{32}$, i vari strumenti di urbanistica partecipata ${ }^{33}$, l'istituto francese (così

26 L. BOBBIO, Dilemmi della democrazia partecipativa, in Dem. e dir., n. 4/2006, p. 12; U. ALLEGRETTI, Democrazia partecipativa: un contributo alla democratizzazione della democrazia, in ID. (a cura di), Democrazia partecipativa. Esperienze e prospettive in Italia e in Europa, Firenze University Press, Firenze, 2010, p. 17; M. DELLA MORTE, Rappresentanza vs. partecipazione? L'equilibrio costituzionale e la sua crisi, FrancoAngeli, Milano, 2012, p. 107. Per una panoramica delle diverse pratiche, ovvero differenti modelli, soggetti, procedure, incidenza, contesti e livelli territoriali, ricondotte e riconducibili alla democrazia partecipativa, cfr. U. ALLEGRETTI, Democrazia partecipativa e processi di democratizzazione, cit., pp. 175 ss. (nonché ID., La democrazia partecipativa in Italia e in Europa, cit.; ID., Il cammino accidentato di un principio costituzionale: quaranta anni di pratiche partecipative in Italia, in Rivista AIC, n. 1/2011).

27 Quanto alla declinazione deliberativa della democrazia, la questione si presenta come più complessa: in estrema sintesi, quest'ultima può configurarsi sia come forma a sé sia come metodo applicabile nel contesto delle altre declinazioni; in tema, fra i classici, J. COHEN, Deliberation and Democratic Legitimacy, in A. HAMLIN, P. PETTIT (eds.), The Good Polity. Normative Analysis of the State, Blackwell, Oxford, 1989; J. HABERMAS, Faktizität und Geltung. Beiträge zur Diskurstheorie des Rechts und des demokratischen Rechtsstaats, trad. it. Fatti e norme. Contributi a una teoria discorsiva del diritto e della democrazia, a cura di L. Ceppa, Guerini e Associati, Milano, 1996, pp. 341 ss.; J. ELSTER (a cura di), Deliberative democracy, Cambridge University Press, Cambridge, 1998; nonché, più recentemente, J. FISHKIN, P. LASLETT (a cura di), Debating Deliberative Democracy, WileyBlackwell, 2003; G. BOSETTI, S. MAFFETTONE (a cura di), Democrazia deliberativa: cosa è, Luiss University Press, Roma, 2004; A. GUTMANN, D. THOMPSON, Why Deliberative Democracy?, Princeton University Press, Princeton, 2004; C. R. SUNSTEIN, Designing Democracy. What Constitutions Do, Oxford University Press, 2001, trad. it. A cosa servono le Costituzioni. Dissenso politico e democrazia deliberativa, il Mulino, Bologna, 2009; A. FLORIDIA, Un'idea deliberativa della democrazia. Genealogia e principi, il mulino, Bologna, 2017; R. BIFULCO, Democrazia deliberativa e principio di realtà, in Federalismi.it, n. 1/2017.

$28 \mathrm{Si}$ intende il termine in senso ampio, come residenti/partecipanti ad una determinata collettività (quindi in una locuzione che include anche gli stranieri).

29 Senza misconoscere ovviamente che la partecipazione è elemento intrinseco ad ogni forma di democrazia: la differenza è nelle modalità della sua estrinsecazione concreta.

30 Sulla democrazia dal basso, cfr. J. BRECHER, T. COSTELLO, B. SMITH, Globalization from Below. The Power of Solidarity, 2000, trad. it. Come farsi un movimento globale. La costruzione della democrazia dal basso, DeriveApprodi, Roma, 2001; A. ALGOSTINO, Democrazia, rappresentanza, partecipazione, cit., pp. 133 ss.

31 A partire dal caso emblematico e noto di Porto Alegre (sul punto esiste ormai una vastissima letteratura: fra i molti cfr. B. DE SOUSA SANTOS, Il bilancio partecipativo a Porto Alegre: per una democrazia redistributiva, e L. AVRITZER, Modelli di deliberazione democratica: un'analisi del bilancio partecipativo, in B. DE SOUSA SANTOS (a cura di), Democratizzare la democrazia. I percorsi della democrazia partecipativa, Città Aperta Edizioni, Troina (En), 2003, pp. 359 ss.; G. ALLEGRETTI, L'insegnamento di Porto Alegre. Autoprogettualità come paradigma urbano, Alinea, Firenze, 2003; M. GRET, Y. SINTOMER, The Porto Alegre Experiment. Learning Lessons for Better Democracy, Zed Books, London, 2005), attraverso l'esperienza spagnola (cfr., per un primo commento, E. GANUZA FERNÁNDEZ, Democrazia e partecipazione: i bilanci partecipativi in Spagna, in Dem. e dir., 3/2006, pp. 70 ss.; ID., Los presupuestos participativos en España: impacto y futuro en la ciudades, in U. ALLEGRETTI (a cura di), Democrazia partecipativa, cit., pp. 113 ss.), per arrivare ai casi italiani (per un primo approccio, cfr. A. L. PECORIELLO, F. RISPOLI, Pratiche di democrazia partecipativa in Italia, in Dem. e dir., n. 3/2006, pp. 115 ss.; V. CANAFOGLIA, Cicli procedurali dei bilanci partecipativi: alcuni esempi italiani, in U. ALLEGRETTI (a cura di), Democrazia partecipativa, cit., pp. 129 ss.); in una prospettiva comparata, cfr. Y. SINTOMER, C. HERZBERG, A. RÖECKE, Les budgets participatifs en Europe, La Découverte, Paris, 2008; Y. SINTOMER, G. ALLEGRETTI, I bilanci partecipativi in Europa. Nuove esperienze democratiche nel nuovo continente, Ediesse, Roma, 2009.

32 Vedi A. RÖECKE, Y. SINTOMER, Estrazione a sorte e democrazia partecipativa: riflessioni sugli esiti delle giurie civiche berlinesi, in Dem. e dir., n. 3/2006, pp. 87 ss.

33 Per un richiamo ad alcune esperienze ed ulteriore bibliografia, cfr. A. L. PECORIELLO, F. RISPOLI, Pratiche di democrazia, cit., spec. pp. 116 ss.; C. CUDIA, La partecipazione ai procedimenti di pianificazione territoriale tra chiunque è interessato, in Dir. pubbl., n. 2008/1, pp. 263 ss.; G. DEPLANO (a cura di), Partecipazione e comunicazione nelle nuove forme del piano urbanistico, Edicom, Monfalcone (GO), 2009; con specifico riferimento alla programmazione sociale territoriale, M. PACI (a cura di), Welfare locale e democrazia partecipativa, il Mulino, Bologna, 2008. 
come di altri Paesi) del débat public ${ }^{34}$, i variforum, consensus building, stakeholder involvement, tavoli ${ }^{35}$.

Si tratta di pratiche eterogenee accomunate, in primo luogo, dall'essere forme di partecipazione atipiche, e, in secondo luogo, dall'essere in qualche modo istituzionalizzate ${ }^{36}$ : in tutte si riscontrano un coinvolgimento delle istituzioni e una formalizzazione da parte del diritto.

Tali elementi comuni conoscono quindi modulazioni differenti: il ruolo esercitato dalle istituzioni si configura come più attivo o passivo, a seconda, ad esempio, della nascita dall'alto o dal basso delle modalità di partecipazione; il recepimento nelle forme del diritto può essere rigido od elastico, prevedere un ambito locale ${ }^{37}$ o nazionale, costituire una semplice sperimentazione, limitarsi ad istituire degli organi o delle sedi di confronto senza una configurazione formale precisa quanto ai loro poteri.

L'esperienza italiana - a livello statale - si configura allo stato essenzialmente come top-down ${ }^{38}$. Mi riferisco al dibattito pubblico sulle grandi opere ${ }^{39}$, previsto dal codice appalti (decreto legislativo n.50 del 2016, art. 22); con la precisazione che, in senso lato, sono stati ricondotti alla democrazia partecipativa anche altri strumenti (come in primis le consultazioni) ${ }^{40}$, alcuni dei quali, come l'accesso civico generalizzato, possono creare canali di comunicazione bottom-up ${ }^{41}$.

Il dibattito pubblico è un meccanismo dominato dalle istituzioni e, in specie,

34 Cfr. C. BLATRIX, L. BLONDIAUX, J.-M. FOURNIAU, B. HÉRIARD DUBREIL, R. LEFEBVRE, M. REVEL (dir.), Le débat public: une expérience française de démocratie partecipative, La Découverte, Paris, 2007; Y. MANSILLON, L'esperienza del «débat public» in Francia, in Dem. e dir., n. 3/2006; M. DELL'OMARINO, Il dibattito pubblico come strumento di democrazia partecipativa. Una breve indagine comparata in occasione della sua introduzione nell'ordinamento italiano, in Diritti comparati. Working Paper, $\mathrm{n}$. 7/2017; C. LOMBARDI, A. LULLO, Il dibattito pubblico quale strumento di democrazia partecipativa (evoluzione e stato dell'arte), in Amministrazione in Cammino, 15 luglio 2018, pp. 15 ss.; sia consentito citare anche A. ALGOSTINO, Democrazia, rappresentanza, partecipazione, cit., pp. 204 ss.

35 Per una panoramica ampia in prospettiva comparata, oltre le opere già citate, cfr. M. H. BACQUÉ, H. REY, Y. SINTOMER (sous la direction de), Gestion de proximité et démocratie participative. Une perspective comparative, La Découverte, Paris, 2005, nonché A. FUNG, E. O. WRIGHT (edited by), Deepening Democracy. Institutional Innovations In Empowered Participatory Governance, Verso, London-New York, 2003; in senso ampio, si vedano altresì A. VALASTRO (a cura di), Le regole della democrazia partecipativa. Itinerari per la costruzione di un metodo di governo, Jovene, Napoli, 2010; D. BOLOGNINO, G. C. DE MARTIN (a cura di), Democrazia partecipativa e nuove prospettive della cittadinanza, Cedam, Padova, 2010; A. RAMIREZ NÀRDIZ, Democracia participativa. La democracia participativa como profundización en la democracia, Tirant lo Blanch, Valencia, 2010.

36 Cfr. M. LUCIANI, Democrazia rappresentativa e democrazia partecipativa, in L. CARLASSARE (a cura di), La sovranità popolare nel pensiero di Esposito, Crisafulli, Paladin, Cedam, Padova, 2004, p. 184); U. ALLEGRETTI, Basi giuridiche della democrazia partecipativa in Italia: alcuni orientamenti, in Dem. e dir., n. 3/2006, p. 159.

37 Con la creazione di "diseguaglianze di/nella democrazia" sul territorio nazionale?

38 Cfr. G. DI GASPARE, Il dibattito pubblico tra democrazia rappresentativa e democrazia partecipativa, in Amministrazione In Cammino, 30 settembre 2017, p. 4, che rileva anche come, invece, a livello locale le procedure siano maggiormente bottom-up.

39 Per grandi opere, come precisa l'allegato 1 al Decreto del Presidente del Consiglio dei Ministri (DPCM) n. 76 del 10 maggio 2018, si intende - citando senza pretesa di completezza ma a titolo esemplificativo - la realizzazione di autostrade o di tronchi ferroviari per un valore di investimento pari o superiore a 500 milioni di euro; porti e terminali marittimi per un valore di investimento superiore a 200 milioni di euro; elettrodotti aerei, con un tracciato di lunghezza superiore a $40 \mathrm{~km}$; insediamenti industriali per investimenti complessivi superiori ai 300 milioni di euro; etc.

40 In senso ampio, sulle consultazioni pubbliche, cfr. P. MARSOCCI, Consultazioni pubbliche e partecipazione popolare, in Rassegna Parlamentare, n. 1/2016, pp. 29 ss.; criticamente, in relazione alla consultazione nell'attività normativa del Governo, A. VALASTRO, Gli istituti di partecipazione, cit., pp. 77 ss.; per le consultazioni a livello statale, si ricorda inoltre l'adozione nel settembre 2017 (XVII legislatura) delle Linee guida per le consultazioni pubbliche promosse dal Senato della Repubblica.

41 Così S. FOÀ, La nuova trasparenza amministrativa, in Diritto amministrativo, n. 1/2017, spec. p. 92. 
dall'esecutivo. Basti qui citare alcuni elementi: 1) il fatto che esso rappresenta meramente una tappa nel procedimento amministrativo ${ }^{42}$; 2) la composizione della Commissione nazionale

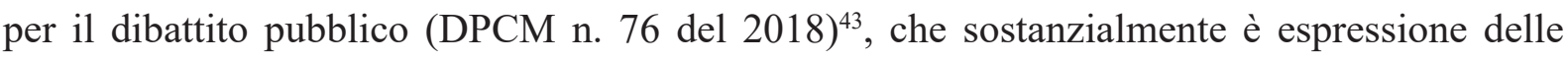
amministrazioni centrali integrate con qualche rappresentanza delle amministrazioni locali, senza alcuna iniezione dal basso, dalla società civile ${ }^{44}$; 3) la gestione (l'indizione e la cura) del dibattito da parte dell' «amministrazione aggiudicatrice» o dell' «ente aggiudicatore» (in quest'ultimo caso, in ipotesi, anche un soggetto privato, il che, peraltro, oltre a non denotare un coinvolgimento dei cittadini, insinua dubbi sull'imparzialità della procedura $)^{45}$.

Nell'insieme "democrazia partecipativa" è poi da sottolineare la valenza differente di strumenti riconducibili alla "semplice" consultazione, con l'ulteriore tipizzazione in relazione al momento della consultazione (in specie, se preventiva o successiva rispetto al processo decisionale), e strumenti attinenti alla partecipazione alla decisione (si pensi all'eventuale valore vincolante attribuito, ad esempio, a scelte inerenti il bilancio partecipativo $)^{46}$.

La declinazione in forma "consultazione" ictu oculi pare carente di efficacia, nonché facilmente incline ad un utilizzo ex parte principis; mentre quella in forma "decisione" presenta inevitabili problemi di raccordo con la democrazia rappresentativa, ovvero tout court con l'eguaglianza politica. Ma riprenderemo dopo il discorso sulle ambiguità della democrazia partecipativa.

Nella concretizzazione italiana a livello statale del dibattito pubblico sulle grandi opere, è da valutare positivamente l'inserimento in una fase iniziale (peraltro in sede di DPCM e senza ulteriori specificazioni) ${ }^{47}$, in coerenza con quanto previsto nella Convenzione di Århus sulla partecipazione dei cittadini in materia ambientale del 1998 (ratificata con legge n. 108 del 2001) e nelle norme dell'Unione europea (direttiva n. 35 del 2003) ${ }^{48}$, lasciando così impregiudicata la realizzabilità di eventuali alternative all'opera, così come la sua stessa effettuazione ${ }^{49}$. È una

42 Sottolinea la contestualizzazione del dibattito all'interno del procedimento amministrativo, con una carenza nella garanzia della partecipazione anche ai sensi della legge n. 241 del 1990, G. DI GASPARE, Il dibattito pubblico, cit., p 6.

43 DPCM n. 76 del 2018, art. 4, c. 1 e 2.

44 Si domanda se il modello italiano non sia troppo legato alla pubblica amministrazione, U. ALLEGRETTI, Un caso di attuazione del principio costituzionale di partecipazione: il regolamento del dibattito pubblico sulle grandi opere, in Rivista AIC, n. 3/2018, p. 470. $45 \mathrm{Si}$ inserisce qui anche la questione inerente il gap, in termini di informazione e risorse, che può esserci fra le parti, con la sproporzione e gli ostacoli che ciò può determinare per una partecipazione effettiva (si pensi, ad esempio, ai costi per l'eventuale intervento di esperti).

46 In tema, si vedano U. ALLEGRETTI, Basi giuridiche, cit., p. 158; Id., Democrazia partecipativa e processi di democratizzazione, cit., pp. 190-191; L. BOBBIO, Dilemmi della democrazia partecipativa, cit., pp. 22-23.

47 DPCM n. 76 del 2018, art. 5; in argomento, cfr. V. MANZETTI, Il "dibattito pubblico" nel nuovo codice contratti, in Federalismi. it, n. 5/2018, pp. 8-9; in senso ampio, sulla (eccessiva) genericità della disposizione legislativa, cfr. G. DI GASPARE, Il dibattito pubblico, cit. pp. 6-7; G. MANFREDI, Il regolamento sul dibattito pubblico: democrazia deliberativa e sindrome nimby, in Urbanistica e appalti, n. 5/2018, p. 605.

48 Convenzione sull'accesso alle informazioni, la partecipazione dei cittadini e l'accesso alla giustizia in materia ambientale, 1998 (legge di autorizzazione alla ratifica 16 marzo 2001, n. 108; direttiva 26 maggio 2003/35/CE del Parlamento europeo e del Consiglio del 26 maggio 2003), art. 6, c. 4.

49 In questo senso, U. ALLEGRETTI, Un caso di attuazione del principio costituzionale, cit., p. 466; si segna in tal modo la differenza rispetto a meccanismi simili, se pur ad hoc, utilizzati in precedenza, come l'Osservatorio per il collegamento ferroviario Torino-Lione (in tema, ci si permette di rinviare a A. ALGOSTINO, L'Osservatorio per il collegamento ferroviario Torino-Lione come case-study sulla democrazia e sul dissenso, in Costituzionalismo.it, n. 2/2009). 
partecipazione i cui esiti - come precisa 1'art. 22, co. 4, del D. Lgs. n. 50 del 2016 - vengono valutati in sede di progettazione definitiva e discussi in sede di conferenza di servizi, dunque non vincolante; il che colloca la forza della partecipazione su un piano, per così dire, deliberativo, ovvero relativo alla ragionevolezza delle proposte, obbligando l'amministrazione ad una (maggior) motivazione in ordine alle proprie scelte ${ }^{50}$. Si precisa che non si intende con quanto appena osservato criticare lo strumento de quo in quanto non dotato di potere decisionale: se così fosse infatti sorgerebbero complicazioni nel raccordo con la democrazia rappresentativa e si rischierebbe di attribuire scelte politiche ad una minoranza, dando vita ad una connotazione elitaria della partecipazione, in violazione del principio di eguaglianza politica e di sovranità popolare.

\section{Democrazia partecipativa fra istanze dal basso e mistificazioni}

Delineato - se pur sinteticamente - il campo di azione della democrazia partecipativa, l'interrogativo è: essa "democratizza" la democrazia?

Innanzitutto una premessa: quanto mai oggi, nell'era della global economic governance, si palesa la necessità di una democratizzazione della sfera economica, mentre - come detto - gli istituti previsti di democrazia partecipativa si inseriscono nel processo decisionale politicoistituzionale.

Ciò precisato, la domanda, se pur ristretta all'ambito istituzionale, resta: la democrazia partecipativa incrementa la "vitalità" della democrazia" ? può costituire un aiuto per superare i limiti strutturali della democrazia rappresentativa, lo stato di asfissia nel quale essa versa, magari realizzando qualcuna delle sue "promesse non mantenute" (Bobbio) ${ }^{52}$ ?

La democrazia spicca - osserva Alfio Mastropaolo - «per la sua smisurata ambizione» e, insieme, per la sua imperfezione, il suo essere «un intrico di conquiste», ma anche di cadute e «drammatiche smentite» ${ }^{53}$. Nell'incompiutezza e, come tutti i fatti umani, reversibilità, della democrazia, la sua declinazione «partecipativa» si inserisce con luci ed ombre.

Le luci: riconoscimento e sviluppo di una cittadinanza attiva, ovvero empowerment

50 Nel DPCM n. 76 del 2018, si precisa che l'amministrazione aggiudicatrice o l'ente aggiudicatore devono evidenziare, nel dossier conclusivo, «le ragioni che hanno condotto a non accogliere eventuali proposte» (art. 7, c. 1, lett. d)).

51 Si veda, ad esempio, L. BLONDIAUX, Le nouvel esprit de la démocratie. Actualité de la démocratie participative, Seuil, Paris, 2008, che, dopo aver analizzato alcune esperienze di democrazie partecipativa contemporanea concludendo con un giudizio «nuancé», propone «les conditions d'une véritable démocratisation de la répresentation par la participation» (p. 101), ovvero le condizioni alle quali i regimi rappresentativi esistenti «meritént effectivement le nom de «démocratie»» (p. 11); in particolare Blondiaux individua tali condizioni in: «prendre au sérieux les formes matérielles de la discussion», «encourager l'émergence de puovoirs neutres», «promouvoir une constitution démocratique mixte», «jouer sur la complémentarité des dispositifs», «repenser la relation à la décision», «réaffirmer sans cesse l'idéal d'inclusion» (pp. 101 ss.).

52 N. BOBBIO, Il futuro della democrazia, Einaudi, Torino, 1983.

53 A. MASTROPAOLO, La democrazia è una causa persa? Paradossi di una invenzione imperfetta, Bollati Boringhieri, Torino, 2011, pp. $7,13$. 
deicittadini $^{54}$, nel segno dell'art. 3 , co. 2 , Cost.; riduzione delle distanze fra chi decide e chi è soggetto alla decisione, con un inveramento della sovranità popolare; possibilità di migliorare qualitativamente le scelte politiche (attraverso il confronto tra saperi e visioni differenti) ${ }^{55}$; maggiore efficienza ed efficacia della decisione (ma qui siamo già in una zona di nuances chiaroscure).

Sono - quelli elencati - possibili effettivi positivi della democrazia partecipativa che, da un lato, si manifestano in direzione della democrazia rappresentativa, innervandola di partecipazione ${ }^{56}$; dall'altro, si incontrano nella relazione con la democrazia dal basso, che può trovare luoghi di comunicazione con le istituzioni nei quali far valere istanze che faticano a trovare ascolto nelle sedi tradizionali.

Ma veniamo alle ombre, procedendo per punti.

1. In primo luogo, in questione è la possibilità che la democrazia partecipativa crei nuovi luoghi di espressione di rivendicazioni sociali ${ }^{57}$ e del pluralismo, dando voce al dissenso e rafforzando il progetto di emancipazione individuale e collettiva ${ }^{58}$; che rappresenti, in altri termini, un piccolo passo in funzione contro-egemonica rispetto alla pervasività e prepotenza del finanzcapitalismo ${ }^{59}$. Il rischio è che le potenzialità si risolvano all'opposto in una sussunzione e un assorbimento delle istanze di rivendicazione e inclusione: non emancipazione e nuovi spazi di manifestazione del dissenso ma cooptazione e assimilazione ${ }^{60}$.

Quanto, ad esempio, il bilancio partecipativo rende i cittadini protagonisti nel cammino verso la "libertà dal bisogno" e quanto, invece, li inganna, imputando a loro stessi la scelta presentata come ineludibile - fra quale bisogno sarà soddisfatto e quale no?

Gli strumenti di partecipazione possono trasfigurarsi in strumenti di "imposizione dolce" ${ }^{61}$, per sostituire - ma solo nella forma - il ricorso alla coercizione (che, in verità, lungi dallo scomparire è incrementato nell'incipit del XXI secolo attraverso le misure repressive

54 «Une citoyenneté plus directement active, et plus largement à un accroissement de l'autonomie des individus dans toutes les sphères de leur existence», per dirlo con le parole di P. ROSANVALLON, La légitimité démocratique, cit., p. 322, che rileva come la riappropriazione sociale della politica costituisce l'immaginario che collega fra loro la teoria di Proudhon, l'esperienza della Comune di Parigi o dei movimenti consiliari degli anni 1918-1920, i progetti di democrazia industriale fra le due guerre e le iniziative dei cittadini degli anni Sessanta.

55 Ragiona di necessità di integrare il sapere politico ed esperto con «le conoscenze esperienziali di chi abita e vive i territori», A. VALASTRO, Gli istituti di partecipazione, cit., p. 41, pp. 70-71.

56 Cfr. G. COTTURRI, La democrazia partecipativa, in Dem. e dir., n. 1/2005, pp. 27 ss.; M. DELLA MORTE, Rappresentanza vs. partecipazione, cit., spec. pp. 32 ss.

57 Cfr. U. ALLEGRETTI, Verso una nuova forma di democrazia: la democrazia partecipativa, in Dem. e dir., n. 3/2006, p. 13, che rileva il legame privilegiato fra i processi di democrazia partecipativa e «finalità redistributive e di giustizia sociale».

58 B. DE SOUSA SANTOS, L. AVRITZER, Per un ampliamento del canone democratico, in B. DE SOUSA SANTOS (a cura di), Democratizzare la democrazia, cit., p. 32, considerano la democrazia partecipativa uno dei «campi sociali e politici in cui, all'inizio del nuovo secolo, si sta reinventando l'emancipazione sociale», attraverso la proposizione di una concezione non egemonica della democrazia (ovvero non identificabile con il modello di «democrazia rappresentativa elitista», p. 22).

59 L. GALLINO, Finanzcapitalismo. La civiltà del denaro in crisi, Einaudi, Torino, 2011.

$60 \mathrm{Su}$ «èlites escludenti», «cooptazione» e «integrazione», si legga B. DE SOUSA SANTOS, L. AVRITZER, Per un ampliamento, cit., p. 37.

61 A. MASTROPAOLO, La democrazia è una causa persa?, cit., p. 334, evidenzia il rischio che le consultazioni siano utilizzate «per mobilitare selettivamente un pubblico circoscritto di cittadini, magari sottraendolo ad altre forme di azione politica e sviandone a proprio vantaggio il potenziale di partecipazione e di protesta». 
del dissenso contenute nella legislazione in tema di sicurezza); non rappresentare, dunque, un luogo di espressione dei conflitti, ma una procedura per anestetizzare i conflitti.

Si legga quanto osserva la Corte costituzionale, che, pronunciandosi sul dibattito pubblico (in una questione riguardante la legge sul tema della Regione Puglia), lo definisce «una fondamentale tappa nel cammino della cultura della partecipazione», un modello di confronto fra la pubblica amministrazione e i soggetti interessati, sottolineando come esso alimenti il dialogo, così da far «emergere eventuali più soddisfacenti soluzioni progettuali»e da disinnescare il conflitto «potenzialmente implicito in qualsiasi intervento che abbia impatto significativo sul territorio» ${ }^{62}$. Non dovrebbe ragionarsi più che di prevenzione e disattivazione del conflitto, del suo riconoscimento ed espressione ${ }^{63}$ ? Non è che interessa, più che favorire la partecipazione, "sgombrare il campo" da potenziali oppositori?

A proposito del dibattito pubblico è stato scritto: «lo svolgimento di un dibattito pubblico consente di prevenire o limitare il c.d. fenomeno Nimby... ossia la contrarietà alla suddetta realizzazione da parte dei soggetti che abitano vicino al luogo dove l'opera dovrebbe venir costruita $\rangle^{64}$. Ora, si può obiettare sia che anche le rivendicazioni Nimby hanno diritto di cittadinanza sia che la lettura presentata è semplicistica: i movimenti territoriali molto spesso veicolano non tanto interessi localistici, bensì visioni del mondo differenti, che, in un contesto democratico, devono avere uno spazio politico nel quale manifestarsi ${ }^{65}$. Altra studiosa pare maggiormente consapevole dell'ampiezza dello scontro presente nei conflitti ambientali, che vengono visti come «caratterizzati da veri e propri conflitti sociali, economici e culturali» ${ }^{66}$, ma inserisce tale considerazione come argomentazione a sostegno dell'utilità del dibattito pubblico nel prevenire tali conflitti, evitando «di ritardare o addirittura di "bloccare" la realizzazione di opere necessarie allo sviluppo e alla ripresa economica» ${ }^{67}$. Si assume come un dato scontato e inoppugnabile il modello sviluppista, nonché l'univocità delle sue estrinsecazioni, e si sottintende che la presenza di opposizioni non costituisce un arricchimento bensì un ostacolo.

La "strumentalizzazione" può avvenire quando la democrazia partecipativa nasca spontaneamente, dal basso, ma più facilmente si manifesta nelle sue forme dall'alto ${ }^{68}$, quando

62 Corte costituzionale, sent. n. 235 del 2018, Cons. dir., par. 6.1; per un primo commento, cfr. P. VIPIANA, Il dibattito pubblico per la prima volta al vaglio della Corte costituzionale, in Forum di Quaderni costituzionali, 25 marzo 2019.

63 Sul legame fra democrazia e conflitto, cfr., per tutti, G. AZZARITI, Diritto e conflitti. Lezioni di diritto costituzionale, Laterza, Roma-Bari, 2010.

64 P. VIPIANA, Il dibattito pubblico, cit., p. 1.

65 A. ALGOSTINO, I movimenti territoriali: una nuova manifestazione del conflitto sociale?, in Parolechiave, 60, Voice, dicembre 2018, pp. 35-47.

66 Una consapevolezza della natura complessa delle opposizioni alle grandi opere è in A. AVERARDI, La decisione amministrativa tra dissenso e partecipazione. Le ragioni del «dibattito pubblico», in Munus, n. 1/2018, pp. 129 ss.

67 V. MANZETTI, Il "dibattito pubblico", cit., p. 4.

68 Cfr. le preoccupazioni espresse anche da chi tendenzialmente valuta con favore l'incremento delle ipotesi di democrazia partecipativa, come L. BOBBIO, Dilemmi della democrazia partecipativa, cit., p. 20; come già accennato, sottolinea «i benefici», ma non dimentica anche i «rischi» della democrazia partecipativa, U. ALLEGRETTI, Democrazia partecipativa e processi di democratizzazione, cit., p. 183; Id., Democrazia partecipativa: un contributo alla democratizzazione della democrazia, cit., pp. 28-29; similmente, mette in luce gli elementi di forza e di debolezza della democrazia partecipativa, L. BLONDIAUX, Le nouvel esprit de la démocratie, cit. 
la volontà manipolatrice può esserne un elemento strutturale ${ }^{69}$.

2. Si affaccia la seconda ombra. Oltre ad evitare il dissenso, la democrazia partecipativa può rappresentare un'ottima operazione di marketing, di strategia di vendita di politiche e decisioni $^{70}$. Nel libro bianco dell'Unione europea, intitolato La governance europea ${ }^{71}$, si rileva come la «partecipazione» tende soprattutto a superare il «sentimento di estraneità rispetto all'azione dell'Unione», che hanno «numerosi Europei», aumentando «la fiducia nel risultato finale e nelle istituzioni da cui emanano» le politiche dell’Unione. Nulla togliendo alle esigenze di miglior comunicazione e informazione, il nodo centrale non dovrebbe essere la ricezione delle istanze provenienti dai cittadini?

Convincere della democrazia o essere democrazia?

Come osserva Alessandra Valastro, a proposito delle riforme costituzionali, ma il discorso può essere esteso, la partecipazione appare «terreno appetibile per alzare il tiro della retorica illudente o mendace $\rangle^{72}$.

Non solo: l'operazione pubblicitaria che si può giocare sulla democrazia partecipativa, può avere come effetto collaterale anche quello di assestare un (altro) colpo ad una democrazia rappresentativa in crisi, denigrandola, attraverso una presentazione a contrario delle virtù della "partecipazione diretta" 73 . Per tacere del fatto, che, spostandosi su un piano sostanziale, in tal modo, da un lato, si svuota ulteriormente il circuito politico-rappresentativo parlamentare, già indebolito dal processo di verticalizzazione del potere nell'esecutivo; dall'altro, si crea una contrapposizione, in chiave plebiscitaria, fra popolo e Parlamento. In questo senso, appare emblematica, nel segno della delegittimazione del Parlamento, la congiunzione fra riduzione del numero dei parlamentari e riforma in discussione dell'art. 71 Cost. ${ }^{74}$.

3. Terza ombra. Un ossimoro: l'immagine della democrazia oligarchica o elitaria ${ }^{75}$. Nonostante si insista sulla natura inclusiva della democrazia partecipativa ${ }^{76}$, sul fatto che essa accordi la partecipazione a tutti e, in particolare, come in molti casi intenda privilegiare interessi

69 Cfr. in tema le considerazioni di C. CROUCH, Postdemocrazia, Laterza, Roma-Bari, 2003 cit., p. 28, a proposito dei focus group politici.

70 CFr. B. DE SOUSA SANTOS, L. AVRITZER, Per un ampliamento, cit., p. 40. G. COTTURRI, La democrazia partecipativa, cit., p. 32, evidenzia fra gli esiti (positivi) della democrazia partecipativa l'assunzione di responsabilità e, parallelamente, l'accettazione anche di sacrifici, sottolineando come essa «abitua alla necessaria commisurazione tra risorse e bisogni»: è cinico e fuorviante interpretare meno positivamente quanto osservato da Cotturri, ovvero, pensare che attraverso la parvenza di una decisione propria la democrazia partecipativa (alcune forme di) abitua a non contestare scelte politiche preconfezionate miranti a smantellare lo Stato sociale?

71 Commissione delle Comunità Europee, Bruxelles, 5.8.2001 (COM (2001) 428 definitivo/2).

72 A. VALASTRO, Gli istituti di partecipazione, cit., p. 40.

$73 \mathrm{Si}$ vedano, in merito, le suggestioni sul rapporto fra società civile, governance e Stato, di A. DENEAULT, «Gouvernance». Le management totalitaire, 2013, trad. it. Governance. Il management totalitario, Neri Pozza, Vicenza, 2018, spec. pp. 27 ss.

74 Sui rischi dell'iniziativa legislativa popolare rafforzata, si vedano, fra gli altri, M. LUCIANI, "Iniziativa legislativa e referendum, le proposte di revisione costituzionale", intervento alla Tavola Rotonda AIC, Roma, 1 marzo 2019, in Osservatorio costituzionale, n. 1-2/2019; A. ANZON DEMMIG, L'iniziativa legislativa popolare "indiretta" (c.d. referendum propositivo) nel progetto di legge costituzionale in itinere, in Forum Quad. Cost., 22 marzo 2019; A. MORRONE, L'iniziativa popolare propositiva: per una democrazia plebiscitaria contro la democrazia rappresentativa?, in Federalismi.it, n. 23/2018.

75 In proposito, si può richiamare anche la democrazia ateniese (L. CANFORA, La democrazia. Storia di un'ideologia, Laterza, Roma-Bari, 2004, spec. pp. 31 ss.).

76 Cfr. U. ALLEGRETTI, La democrazia partecipativa in Italia e in Europa, cit., pp. 8-9. 
o posizioni "deboli"77 (potendosi in tal caso - si può aggiungere - ragionare di "disuguaglianza" inscritta nella prospettiva dell'eguaglianza sostanziale), nessuno dei vari meccanismi utilizzati per scegliere i partecipanti ad una pratica di democrazia partecipativa è in grado di ovviare al rischio di creare ipoteticamente una "democrazia perfetta", ma per pochi ${ }^{78}$, ovvero di scivolare verso forme oligarchiche ed elitarie di "governo dei migliori”. Si profila il rischio, ed il discorso si può estendere, più ampiamente, alla retorica della società civile, che negli strumenti di democrazia partecipativa si annidi un approccio antiegualitario, ovvero si tratti di una partecipazione, per così dire, "radical-chic", e, dunque, non si produca, ad esempio, una effettiva emancipazione dei ceti più svantaggiati, ma si possa instaurare una forma più raffinata di esclusione, magari nel loro nome.

4. Quarta ombra: lo spettro del "cittadino totale"79 , di una partecipazione monopolizzante, in un orizzonte in cui gli individui sono asserviti all'interesse, artificialmente presentato come univoco, della polis. E il "cittadino totale" - citando Bobbio - «non è a ben guardare che l'altra faccia non meno minacciosa dello stato totale ${ }^{80}$.

5. Quinta ombra: l'atomizzazione, delle persone e degli interessi. Da un lato, il rischio è di implementare la costruzione di una società civile composta da tanti singoli ${ }^{81}$, nella logica dell'individuo imprenditore di se stesso $^{82}$, alimentando la vulgata del superamento delle classi sociali ${ }^{83}$ e disperdendo la forza delle organizzazioni collettive; dall'altro, il pericolo è la frammentazione degli interessi e degli interventi, con l'abbandono dell'orizzonte della scelta politica capace di situare i singoli interessi settoriali all'interno di una visione del mondo.

\section{Osservazioni conclusive tra immaginazione e realtà}

Venendo alle conclusioni, vorrei riprendere in particolare un punto, nella prospettiva di una demistificazione costruttiva, attenta alla configurazione reale dei rapporti di forza, ma

77 U. ALLEGRETTI, Democrazia partecipativa e processi di democratizzazione, cit., pp. 188-189.

$78 \mathrm{Sul}$ paradosso per cui la democrazia partecipativa «ambisce a includere tutti, ma - di fatto - riesce a coinvolgere concretamente solo qualcuno», vedi L. BOBBIO, Dilemmi della democrazia partecipativa, cit., pp. 14 ss., il quale analizza criticamente i metodi più utilizzati per democratizzarla (la porta aperta, il microcosmo come punti di vista e il microcosmo come campione casuale).

79 R. DAHRENDORF, Cittadini e partecipazione: al di là della democrazia rappresentativa?, in Il cittadino totale. Partecipazione, eguaglianza e libertà nelle democrazie d'oggi, Biblioteca della Libertà, Torino, 1977, pp. 33 ss.

80 N. BOBBIO, Il futuro della democrazia, cit., p. 35: «il cittadino totale e lo stato totale sono le due facce della stessa medaglia, perché hanno in comune, se pur una volta considerato dal punto di vista del popolo, l'altra volta dal punto di vista del principe, lo stesso principio: che tutto è politica, ovvero la riduzione di tutti gli interessi umani agli interessi della polis, la politicizzazione integrale dell'uomo...».

81 Come osserva M. MANETTI, Costituzione, partecipazione democratica, populismo, cit., p. 385, «andrebbe considerata la possibilità che l'espansione virtualmente illimitata dei diritti individuali abbia rappresentato un risvolto del progetto neo-liberale, dove le istanze di partecipazione alla vita politica, ovvero di ricerca della felicità collettiva, sarebbero state incanalate verso la felicità individuale».

82 Interessanti considerazioni in merito, e ulteriori rinvii bibliografici, sono in F. PIZZOLATO, Mutazioni del potere economico e nuove immagini della libertà, in Costituzionalismo.it, n. 3/2017.

83 Sull'esistenza delle classi sociali, nonostante l'oscuramento da parte dei maître à penser dominanti, o la loro minore visibilità (ovvero, in termini marxisti, la loro esistenza in sé ma non per sé), si vedano L. GALLINO, La lotta di classe dopo la lotta di classe, intervista a cura di P. BORGNA, Laterza, Roma-Bari, 2012; M. TRONTI, Lavoro, in Democrazia e diritto, nn. 1-2/2012, pp. 10-11; J. HOLLOWAY, Che fine ha fatto la lotta di classe?, manifestolibri, Roma, 2007; D. LOSURDO, La lotta di classe. Una storia politica e filosofica, Laterza, Roma-Bari, 2013. 
nello stesso tempo aperta ad una fantasia proiettata verso il futuro. Mi pare che centrale sia la considerazione di quanto la democrazia partecipativa sia vicina alle possibilità di espressione del dissenso e del conflitto e quanto all'efficacia del comando e ad una concertazione-cooptazione.

Se, concretizzando il discorso in relazione all'istituto che, per antonomasia, impersonifica la democrazia partecipativa a livello statale, «la finalità del dibattito pubblico è quella di democratizzare e legittimare la decisione a venire, in modo che, seppure non accettata da tutti, risulti accettabile, perché tutti sono stati ascoltati» (Mansillon) ${ }^{84}$, non si può che dubitare dello strumento: è un arricchimento della democrazia prevenire la formazione di dissenso attraverso un ascolto incanalato e finalizzato all'accettabilità di una decisione?

Si riducono gli spazi di discussione nella democrazia rappresentativa, sempre più autoreferenziale e allo stesso tempo etero-diretta ${ }^{85}$, e si aprono spazi di consultazione e concertazione: come non pensare ad un loro utilizzo in chiave plebiscitaria? Alla creazione di una - utilizzando l'espressione di Marcuse - «confortevole, levigata, ragionevole, democratica non-libertà» ${ }^{86}$ ?

Per una partecipativa effettiva si rivela essenziale la sua configurazione in senso controegemonico, come spazio per il conflitto, e, in questa prospettiva, è fondamentale immaginare strumenti di partecipazione che agiscano, per aprire almeno qualche piccola crepa nell'egemonia del neoliberismo, nella sfera dell'economia, coniugando, per ragionare nei termini della Costituzione italiana, una ri-vitalizzazione dell'articolo 41 (con la possibilità di limitare nonché di indirizzare e coordinare l'iniziativa economica privata) a concretizzazioni degli articoli 43 e 46 (che aprono a prospettive di nazionalizzazioni, di collaborazione dei lavoratori e di affidamenti di imprese a comunità di lavoratori e cittadini), nell'orizzonte dell'art. 3 , co. $2^{87}$. Non è solo teoria: vi è l'esempio concreto delle fabbriche recuperate e autogestite, ma anche tentativi come quello di ri-pubblicizzare l'acqua, prevedendo nelle strutture di gestione forme di partecipazione dei cittadini, che si affianchino, collaborando e non surrogandolo, al circuito rappresentativo degli enti locali.

Infine, un'ultima precisazione, perché gli strumenti di democrazia partecipativa sfuggano alle ombre e consentano, come propone Eduardo Galeano, di "delirar por un ratito" e "adivinar otro mundo posible" ${ }^{88}$, è essenziale il ruolo della democrazia dal basso, dei movimenti sociali, dell'associazionismo, ovvero dei soggetti che oggi si stanno facendo carico del progetto di attuazione della Costituzione ${ }^{89}$, rivendicando emancipazione e "essendo" emancipazione, nel

84 Y. MANSILLON, L'esperienza del «débat public», cit., p. 107.

85 Il riferimento è all'influenza delle decisioni assunte altrove, in specie nella nebulosa della global economic governance.

86 Così l'incipit di H. MARCUSE, One-Dimensional Man. Studies in the Ideology of Advanced Industrial Society, Beacon Press, Boston, 1964, trad. it. L'uomo a una dimensione. L'ideologia della società industriale avanzata, Einaudi, Torino, 1999 , p. 15.

87 Sulla riconduzione degli strumenti partecipativi all'attuazione costituzionale, cfr. A. VALASTRO, Gli istituti di partecipazione, cit., p. 74.

88 E. GALEANO, El derecho al delirio, 1998 ((pubblicato in Patas arriba. La escuela del mundo al revés, Siglo XXI, Madrid, 1998). 89 In argomento, ci si permette di rinviare a A. ALGOSTINO, Settant'anni di "uso" della Costituzione: da patto sociale a progetto alternativo? Brevi note per un contributo al seminario di Costituzionalismo.it, in Costituzionalismo.it, n. 2/2018, pp. 123 ss. 
segno di quella che Abensour definisce "democrazia insorgente"

La convivenza fra le varie forme della democrazia (rappresentativa, diretta, partecipativa, dal basso), attraverso la complementarietà dei processi di intermediazione e composizione politico-partitica, dei canali istituzionali di partecipazione e dei fenomeni di auto-organizzazione, può dare una nuova linfa al costituzionalismo, nel suo tentativo di limitare il potere nel nome della persona e dei suoi bisogni, edificando una casa rispettosa del pluralismo.

\section{Riferimenti bibliografici}

ABENSOUR, M., La Démocratie contre l'État. Marx et le moment machiavélien, 2004, trad. it. La democrazia contro lo Stato. Marx e il momento machiavelliano, Cronopio, Napoli, 2008.

ALGOSTINO, A., Democrazia, rappresentanza, partecipazione. Il caso del movimento No Tav, Jovene, Napoli, 2011.

, I movimenti territoriali: una nuova manifestazione del conflitto sociale?, in Parolechiave, 60, Voice, dicembre 2018.

, L'Osservatorio per il collegamento ferroviario Torino-Lione come case-study sulla democrazia e sul dissenso, in Costituzionalismo.it, n. 2/2009.

, Settant'anni di “uso" della Costituzione: da patto sociale a progetto alternativo?

Brevi note per un contributo al seminario di Costituzionalismo.it, in Costituzionalismo.it, n. $2 / 2018$.

ALLEGRETTI, G., L'insegnamento di Porto Alegre. Autoprogettualità come paradigma urbano, Alinea, Firenze, 2003; M. GRET, Y. SINTOMER, The Porto Alegre Experiment. Learning Lessons for Better Democracy, Zed Books, London, 2005.

, Politiche di partecipazione in Venezuela: l'inizio di un percorso di statuizione normativa, in Dem. e dir., n. 4/2006.

, Basi giuridiche della democrazia partecipativa in Italia: alcuni orientamenti, in

Dem. e dir., n. 3/2006.

, Democrazia partecipativa e processi di democratizzazione, in Dem. e dir., n. $2 / 2008$

, Democrazia partecipativa: un contributo alla democratizzazione della democrazia, in ID. (a cura di), Democrazia partecipativa. Esperienze e prospettive in Italia e in Europa, Firenze University Press, Firenze, 2010.

, Il cammino accidentato di un principio costituzionale: quaranta anni di pratiche

90 Cfr. M. ABENSOUR, La Démocratie contre l'État. Marx et le moment machiavélien, 2004, trad. it. La democrazia contro lo Stato. Marx e il momento machiavelliano, Cronopio, Napoli, 2008. 
partecipative in Italia, in Rivista AIC, n. 1/2011.

, Il Movimento internazionale come attore costituzionale, in Dem. e dir., n. $1 / 2004$

, La democrazia partecipativa in Italia e in Europa, in Rivista AIC, n. 1/2011.

, Un caso di attuazione del principio costituzionale di partecipazione: il regolamento del dibattito pubblico sulle grandi opere, in Rivista AIC, n. 3/2018.

, Verso una nuova forma di democrazia: la democrazia partecipativa, in Dem. e dir., n. 3/2006.

ANZON DEMMIG, A., L'iniziativa legislativa popolare "indiretta" (c.d. referendum propositivo) nel progetto di legge costituzionale in itinere, in Forum Quad. Cost., 22 marzo 2019.

ARIENZO, A., Dalla corporate governance alla categoria politica di governance, in G. BORRELLI (a cura di), Governance, Dante\&Descartes, Napoli, 2004.

AVERARDI, A., La decisione amministrativa tra dissenso e partecipazione. Le ragioni del «dibattito pubblico», in Munus, n. 1/2018.

AVRITZER, L., Modelli di deliberazione democratica: un'analisi del bilancio partecipativo, in B. DE SOUSA SANTOS (a cura di), Democratizzare la democrazia. I percorsi della democrazia partecipativa, Città Aperta Edizioni, Troina (En), 2003.

AZZARITI, G., Democrazia partecipativa: cultura giuridica e dinamiche istituzionali, in Costituzionalismo.it, n. 3/2009.

AZZARITI, G., Diritto e conflitti. Lezioni di diritto costituzionale, Laterza, Roma-Bari, 2010.

BACQUÉ, M. H., REY, H., SINTOMER, Y. (sous la direction de), Gestion de proximité et démocratie participative. Une perspective comparative, La Découverte, Paris, 2005.

BASSO, L., Per uno sviluppo democratico nell'ordinamento costituzionale italiano, in Studi per il ventesimo anniversario dell'Assemblea costituente, v. IV: Aspetti del sistema costituzionale, Firenze, 1969.

BIFULCO, P., Democrazia deliberativa e principio di realtà, in Federalismi.it, n. 1/2017.

BLATRIX, C., BLONDIAUX, L., FOURNIAU, J.-M., HÉRIARD DUBREIL, B., LEFEBVRE, R., REVEL, M. (dir.), Le débat public: une expérience française de démocratie partecipative, La Découverte, Paris, 2007.

BLONDIAUX, L., Le nouvel esprit de la démocratie. Actualité de la démocratie participative, Seuil, Paris, 2008.

BOBBIO, L., Dilemmi della democrazia partecipativa, in Dem. e dir., n. 4/2006.

BOBBIO, N., Il futuro della democrazia, Einaudi, Torino, 1983. 
BOLOGNINO, D., DE MARTIN, G. C. (a cura di), Democrazia partecipativa e nuove prospettive della cittadinanza, Cedam, Padova, 2010.

BOSETTI, G., MAFFETTONE, S. (a cura di), Democrazia deliberativa: cosa è, Luiss University Press, Roma, 2004.

BRECHER, J., T. COSTELlO, B. SMITH, Globalization from Below. The Power of Solidarity, 2000, trad. it. Come farsi un movimento globale. La costruzione della democrazia dal basso, DeriveApprodi, Roma, 2001.

CANFORA, L., La democrazia. Storia di un'ideologia, Laterza, Roma-Bari, 2004.

CARAVITA, B., Oltre l'eguaglianza formale. Un'analisi dell'art. 3 comma 2 della Costituzione, Padova, 1984.

COHEN, J., Deliberation and Democratic Legitimacy, in A. HAMLIN, P. PETTIT (eds.), The Good Polity. Normative Analysis of the State, Blackwell, Oxford, 1989.

COTTURRI, G., La democrazia partecipativa, in Dem. e dir., n. 1/2005.

CRISAFULLI, V., La sovranità popolare nella Costituzione italiana, in Rassegna Giuliana di Diritto e Giurisprudenza, 1954.

CROUCH, C., Postdemocrazia, Laterza, Roma-Bari, 2003.

CUDIA, C., La partecipazione ai procedimenti di pianificazione territoriale tra chiunque è interessato, in Dir. pubbl., n. 2008/1.

D'ALBERGO, S., Dalla democrazia sociale alla democrazia costituzionale (un percorso dell'ideologia giuridica), in Costituzionalismo.it, n. 3/2005.

DAHRENDORF, R., Cittadini e partecipazione: al di là della democrazia rappresentativa?, in Il cittadino totale. Partecipazione, eguaglianza e libertà nelle democrazie d'oggi, Biblioteca della Libertà, Torino, 1977.

DE SOUSA SANTOS, B., Il bilancio partecipativo a Porto Alegre: per una democrazia redistributiva, in B. De Sousa Santos (a cura di), Democratizzare la democrazia. I percorsi della democrazia partecipativa, Città Aperta Edizioni, Troina (En), 2003.

DE SOUSA SANTOS, B., AVRITZER, L., Per un ampliamento del canone democratico, in B. DE SOUSA SANTOS (a cura di), Democratizzare la democrazia. I percorsi della democrazia partecipativa, Città Aperta Edizioni, Troina (En), 2003.

DELL'OMARINO, M., Il dibattito pubblico come strumento di democrazia partecipativa. Una breve indagine comparata in occasione della sua introduzione nell'ordinamento italiano, in Diritti comparati. Working Paper, n. 7/2017.

DELLA MORTE, M., Rappresentanza vs. partecipazione? L'equilibrio costituzionale e la sua crisi, FrancoAngeli, Milano, 2012.

DENEAULT, A., «Gouvernance». Le management totalitaire, 2013, trad. it. Governance. 
Il management totalitario, Neri Pozza, Vicenza, 2018.

DEPLANO, G. (a cura di), Partecipazione e comunicazione nelle nuove forme del piano urbanistico, Edicom, Monfalcone (GO), 2009.

DI GASPARE, G., Il dibattito pubblico tra democrazia rappresentativa e democrazia partecipativa, in Amministrazione In Cammino, 30 settembre 2017.

DOGLIANI, M., GIORGI, C., Costituzione italiana: art. 3, Carocci, Roma, 2017.

ELSTER, J. (a cura di), Deliberative democracy, Cambridge University Press, Cambridge, 1998.

FERRAJOLI, L., Principia iuris. Teoria del diritto e della democrazia, 2. Teoria della democrazia, Laterza, Roma.Bari, 2007.

FERRARA, G., La crisi del neoliberismoe della governabilità coatta, in Costituzionalismo. it, n. $1 / 2013$.

, La sovranità popolare e le sue forme, in S. LABRIOLA (a cura di), Valori e principi del regime repubblicano, 1.I Sovranità e democrazia, Laterza, Roma-Bari, 2006.

FERRARESE, M. R., La governance tra politica e diritto, il Mulino, Bologna, 2010.

FISHKIN, J., LASLETT, P. (a cura di), Debating Deliberative Democracy, WileyBlackwell, 2003.

FLORIDIA, A., Un'idea deliberativa della democrazia. Genealogia e principi, il mulino, Bologna, 2017.

FOÀ, S., La nuova trasparenza amministrativa, in Diritto amministrativo, n. 1/2017.

FUNG, A., WRIGHT, E. O. (edited by), Deepening Democracy. Institutional Innovations In Empowered Participatory Governance, Verso, London-New York, 2003.

GALLINO, L., Finanzcapitalismo. La civiltà del denaro in crisi, Einaudi, Torino, 2011. , La lotta di classe dopo la lotta di classe, intervista a cura di P. BORGNA, Laterza, Roma-Bari, 2012.

GUTMANN, A., THOMPSON, D., Why Deliberative Democracy?, Princeton University Press, Princeton, 2004.

HABERMAS, J., Faktizität und Geltung. Beiträge zur Diskurstheorie des Rechts und des demokratischen Rechtsstaats, trad. it. Fatti e norme. Contributi a una teoria discorsiva del diritto e della democrazia, a cura di L. Ceppa, Guerini e Associati, Milano, 1996.

HELD, D., Models of Democracy, 1996, trad. it. Modelli di democrazia, il Mulino, Bologna, 1997.

HOLLOWAY, J., Che fine ha fatto la lotta di classe?, manifestolibri, Roma, 2007.

LOMBARDI, C., LULLO, A., Il dibattito pubblico quale strumento di democrazia partecipativa (evoluzione e stato dell'arte), in Amministrazione in Cammino, 15 luglio 2018. 
LOSURDO, D., La lotta di classe. Una storia politica e filosofica, Laterza, Roma-Bari, 2013

LUCIANI, M., "Iniziativa legislativa e referendum, le proposte di revisione costituzionale", intervento alla Tavola Rotonda AIC, Roma, 1 marzo 2019, in Osservatorio costituzionale, n. 1-2/2019.

, Democrazia rappresentativa e democrazia partecipativa, in L. CARLASSARE (a cura di), La sovranità popolare nel pensiero di Esposito, Crisafulli, Paladin, Cedam, Padova, 2004.

, Radici e conseguenze della scelta repubblicana di fondare la Repubblica democratica sul lavoro, in ADL - Argomenti dir. lavoro, 3/2010.

LUXEMBURG, R., La rivoluzione russa, 1918.

MANETTI, M., Costituzione, partecipazione democratica, populismo, in Rivista AIC, n. $3 / 2018$.

MANFREDI, G., Il regolamento sul dibattito pubblico: democrazia deliberativa e sindrome nimby, in Urbanistica e appalti, n. 5/2018.

MANSILLON, Y., L'esperienza del «débat public» in Francia, in Dem. e dir., n. 3/2006.

MANZETTI, V., Il "dibattito pubblico" nel nuovo codice contratti, in Federalismi.it, n. $5 / 2018$.

MARSOCCI, P., Consultazioni pubbliche e partecipazione popolare, in Rassegna Parlamentare, n. 1/2016.

MARTINES, T., Art. 56-58, in G. BRANCA (a cura di), Commentario della Costituzione, Le Camere, Tomo I, Bologna-Roma, 1984.

MASTROPAOLO, A., La democrazia è una causa persa? Paradossi di una invenzione imperfetta, Bollati Boringhieri, Torino, 2011.

MESSINA, G., Diritto liquido? La governance come nuovo paradigma della politica e del diritto, Franco Angeli, Milano, 2012.

MORRONE, A., L'iniziativa popolare propositiva: per una democrazia plebiscitaria contro la democrazia rappresentativa?, in Federalismi.it, n. 23/2018.

MORTATI, C., Art. 1, in G. BRANCA (a cura di), Commentario della Costituzione, Principi fondamentali, Bologna-Roma, 1957.

, Istituzioni di diritto pubblico, I, X ed. rielaborata ed aggiornata a cura di F. Modugno, A. Baldassarre e C. Mezzanotte, Cedam, Padova, 1991.

NOCILLA, D., Popolo (dir. cost.), in Enc. dir., XXXIV, Giuffrè, Milano, 1985.

PACI, M. (a cura di), Welfare locale e democrazia partecipativa, il Mulino, Bologna, 2008. 
PATEMAN, C., Participation and democratic theory, Cambridge University Press, 1970.

PECORIELLO, A. L., RISPOLI, F., Pratiche di democrazia partecipativa in Italia, in Dem. e dir., n. 3/2006.

PICCHI, M., Il diritto di partecipazione: note preliminari (per l'effettività dei diritti sociali), nella rivista del Gruppo di Pisa, n. 3/2012.

PICCIONE, D., Presidenzialismo e democrazia partecipativa nella Costituzione bolivariana del Venezuela, in Dir. pubbl. comp. ed europeo, n. 2005-I.

PIZZOLATO, F., Mutazioni del potere economico e nuove immagini della libertà, in Costituzionalismo.it, n. 3/2017.

RAMIREZ NÀRDIZ, A., Democracia participativa. La democracia participativa como profundización en la democracia, Tirant lo Blanch, Valencia, 2010.

RÖECKE, A., SINTOMER, Y., Estrazione a sorte e democrazia partecipativa: riflessioni sugli esiti delle giurie civiche berlinesi, in Dem. e dir., n. 3/2006.

ROSANVALLON, P., La légitimité démocratique. Impartialité, réflexivité, proximité, Seuil, Paris, 2008.

SINTOMER, Y., ALLEGRETTI, G., I bilanci partecipativi in Europa. Nuove esperienze democratiche nel nuovo continente, Ediesse, Roma, 2009.

SINTOMER, Y., HERZBERG, C., RÖECKE, A., Les budgets participatifs en Europe, La Découverte, Paris, 2008.

SUNSTEIN, C. R., Designing Democracy. What Constitutions Do, Oxford University Press, 2001, trad. it. A cosa servono le Costituzioni. Dissenso politico e democrazia deliberativa, il Mulino, Bologna, 2009.

TOSATO, E., Sovranità del popolo e sovranità dello Stato, in Riv. Trim. Dir. Pubbl., 1957.

TRONTI, M., Lavoro, in Democrazia e diritto, nn. 1-2/2012.

VALASTRO, A., (a cura di), Le regole della democrazia partecipativa. Itinerari per la costruzione di un metodo di governo, Jovene, Napoli, 2010.

, Gli istituti di partecipazione fra retorica delle riforme e umiltà dell'attuazione, in Costitutizonalismo.it, n. 1/2017.

VIPIANA, P., Il dibattito pubblico per la prima volta al vaglio della Corte costituzionale, in Forum di Quaderni costituzionali, 25 marzo 2019. 


\section{SOBRE A AUTORA:}

Alessandra Algostino é doutora em Direito pela Università degli Studi di Ferrara e professora de Direito Constitucional da Università degli Studi di Torino. É autora de L'ambigua universalità dei diritti. Diritti occidentali o diritti della persona umana? (Jovene, 2005), I diritti politici dello straniero (Jovene, 2006), Democrazia, rappresentanza, partecipazione. Il caso del movimento No Tav (Jovene, 2011), Diritto proteiforme e conflitto sul diritto, Studio sulla trasformazione delle fonti del diritto (Giappichelli, 2018), dentre outros textos. Em seus temas de pesquisa incluemse: direito, migração, trabalho, democracia, participação e movimentos, fontes do direito, relação entre direito e economia.

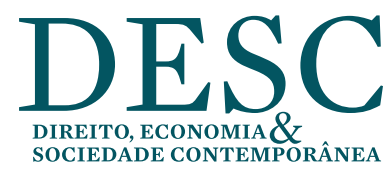

